\title{
Assessment of hand-transmitted vibration in self propelled vertical conveyor reaper
}

\author{
T.R. Gururaj and A.K. Mehta
}

Received : 10.03.2020; Revised : 08.08.2020; Accepted : 07.09.2020

See end of the Paper for authors' affiliation

Correspondence to :

T.R. Gururaj

GPS Institute of Agricultural

Management, Bengaluru

(Karnataka) India

Email: gururajsuni@gmail.com
- ABSTRACT : The self propelled vertical conveyor reaper is commonly used for harvesting wheat, rice etc. It has become a main or the sole of mechanical power source on small and medium size farms in India. The operators of VCR are exposed to a high level of vibration arising from single cylinder engine during field operations. The vibration from the VCR is transmitted from handle to hands, arms and shoulders. The detrimental effect of the prolonged exposure to hand-transmitted vibration on the operators has been known for a long time. In the present study, experiments were conducted to assess the vibration extent in VCR for two operational conditions i.e. transportation on bitumen road and wheat harvesting operation. The vibrations were measured at engine speed 2200 and $2800 \mathrm{rpm}$. In this study it was found that the vibration magnitudes decreased with increase in engine speed from 2200 to $2800 \mathrm{rpm}$ in both operational conditions. The highest vibration values were observed in $\mathrm{x}$-direction. The maximum frequency-weighted vibration acceleration (rms) in $\mathrm{x}$-direction was 18.76 and $22.8 \mathrm{~ms}^{-2}$ in transportation and wheat harvesting. One third octave band frequency spectra were also obtained. The peak acceleration appeared around $50 \mathrm{~Hz}$ for both transportation and wheat harvesting at engine speed 2200 and $2800 \mathrm{rpm}$. The average 8 hour exposure time for occurrence of white finger syndrome was 1.16 and 0.93 years at transportation and wheat harvesting operation.

- KEY WORDS : VCR, Engine speed, Wheat harvesting, Vibrations, White finger syndrome

- HOW TO CITE THIS PAPER : Gururaj, T.R. and Mehta, A.K. (2020). Assessment of handtransmitted vibration in self propelled vertical conveyor reaper. Internat. J. Agric. Engg., 13(2) : 167-174, DOI: 10.15740/HAS/IJAE/13.2/167-174. Copyright@2020: Hind Agri-Horticultural Society. 